\title{
Enhancement of energy consumption estimation for electric vehicles by using machine learning
}

\author{
Adnane Cabani ${ }^{1}$, Peiwen Zhang ${ }^{2}$, Redouane Khemmar ${ }^{3}$, Jin $\mathrm{Xu}^{4}$ \\ 1,2,3 Normandie Univ, UNIROUEN, ESIGELEC, IRSEEM, 76000 Rouen, France \\ ${ }^{4}$ École de Technologie Supérieure, University of Quebec, Montreal, QC, H3C 1K3, Canada
}

\begin{abstract}
Article Info
Article history:

Received Apr 21, 2020

Revised Jan 25, 2021

Accepted Feb 7, 2021

\section{Keywords:}

Electric vehicle

Energy consumption

Intelligent transportation

systems

$\mathrm{K}-\mathrm{NN}$

Machine learning

ABSTRACT

Three main classes are considered of significant influence factors when predicting the energy consumption rate of electric vehicles (EV): environment, driver behaviour, and vehicle. These classes take into account constant or variable parameters which influences the energy consumption of the EV. In this paper, we develop a new model taking into account the three classes as well as the interaction between them in order to improve the quality of EV energy consumption. The model depends on a new approach based on machine learning and especially $k-\mathrm{NN}$ algorithm in order to estimate the EV energy consumption. Following a lazy learning paradigm, this approach allows better estimation performance. The advantage of our proposal, in regards to mathematical approach, is taking into account the real situation of the ecosystem on the basis of historical data. In fact, the behavior of the driver (driving style, heating usage, air conditioner usage, and battery state) impacts directly the EV energy consumption. The obtained results show that we can reach up to $96.5 \%$ of accuracy about the estimated of energy-consumption. The proposed method is used in order to find the optimal path between two points (departure-destination) in terms of energy consumption.
\end{abstract}

This is an open access article under the CC BY-SA license.

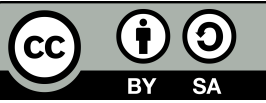

\section{Corresponding Author:}

Adnane Cabani ESIGELEC, IRSEEM

Rouen Madrillet Innovation, Avenue Galilée-BP 10024, 76801 Saint-Etienne du Rouvray Cedex, France Email: cabani@esigelec.fr

\section{INTRODUCTION}

Global warming, an international treaty to reduce greenhouse gas emissions has emerged: it is the Kyoto protocol. The commitments made are binding on the different signatory countries. One of the key commitments is to reduce emissions of these gases by at least $5 \%$ from the 1990 level for 2012 . Faced with this problem, the European Union has adopted ' $3 \times 20$ ' objectives by 2020 : i) Increase to $20 \%$ the share of renewable energies, ii) Reducing CO2 emissions by $20 \%$ compared to 1990 , and iii) Increase energy efficiency by $20 \%$.

In order to achieve these objectives, various stakeholders are working to propose different solutions for the transport of people, among other things. In this way, as we know that drivers waste several minutes in finding a parking place, the authors of [1] were interested in proposing a model of car parking assistance system using camera networks in order to reduce the ecological footprint. Other work aims to improve traffic flow while reducing the impact on the environment by removal toll plazas [2-4]. These work gave rise to the pre-standard [5]. For the case of electric vehicles, the authors of $[6,7]$ have proposed an intelligent navigation systembased optimization of the energy consumption. Authors have used a proprioceptive and exteroceptive sensors in order to propose to drivers the optimal path based energy consumption. It is a mathematical modelling of the 
vehicle's behaviour and the discharge of the battery. The personal behaviors of driver and their impacts were not considered in terms of energy consumption. However, we know the behavior of battery discharge depends on many parameters. The same vehicle equipped with the same type of battery may behave completely different depending on where the vehicle travels (countries with snow, cold or hot weather), whether the vehicle sleeps outside or inside a garage, type of driving and the use of the vehicle's accessories (listening to the radio, turning on the heating or air conditioner, and recharge mode (fast or slow). All these parameters are difficult to model. Today, we know that drivers complain that estimated charge battery presented in the dashboard of electric vehicle are mostly time inaccurate.

In the literature, many studies have been proposed for the prediction of range to improve the efficient use of electric vehicles (EVs) and in which they take into account single factor that influence EV range. In [8], a interesting presentation and study of the different issues and challenges for EVs range prediction have been introduced. The analysis was carried out by the study of the impact of different factors which has a significant correlation with the range estimation. The study takes into account three major classes of significant factors: The vehicle design, the driver, and the environment. Significant parameters were identified like constant parameters (vehicle type, transmission, mass, weight, battery type, charging station, and road.) and variable parameters (battery state of charge and battery state of health [9], driver behavior [10], traffic flow [11], and external environment factors [12,13]). The proposal introduced in [8] concludes that the EV's range prediction is currently, and in most cases, based on state of charge estimation of the battery pack (54\%). The ambient temperature (25\%), driver behavior (10\%), and route (6\%) comes after but still significant too. In [14], another approach which studied the impact of the driver in the EVs consumption is to use data-driven for driver's behavior modelling. Authors present a good way to increase the acceptance of driver assistance systems by adapting them to both needs and behavior of the driver. For this, the authors use vehicle data combined to probabilistic affiliation of the driver (anxious, and aggressive.). An anticipatory energy saving assistant (ANESA) [14] was developed for giving driving hints/instruction for reducing energy consumption specifically in tight curves. This is done through a probabilistic map including possible destinations in which both of state of charge (SoC) parameter and EV's energy consumption were considered as random variables. This approach uses an inverse reinforcement learning method from [15]. In [15], the authors present an innovative approache which combined several kinds of parameters (EV parameters, road conditions, traffic, weather, and historical driver behavior.) to determine the range autonomy with high accuracy. The estimation of the EV range is based on data mining with regression model. Today, models which combine multiple factors in a single model present more and more efficient results. Different approaches are taken into account the topology of the road as an impact factor to reduce the energy consumption. One way to combine different factors is to assign them to a discrete segment of road like Google Maps [8]. In [16], the road network is considered as a graph in which each segment includes different factors that influence the EV's consumption like traffic, air temperature, and climbing of descending angle. In [17], the authors have extended the algorithm in order to handle negative cycle costs which are caused by the energy recuperation. The algorithm produced for each road segment a cost function which is equal to the amount of energy necessary to carry out the segment road. The graph representation presents high efficient even it does not take into account the driver behavior [16, 18]. In [19], new model-based approach for predicting remaining driving range (RDR) was developed in which we can find a combination between both particle filter and Markov chains. The RDR prediction is represented as a probability distribution approximated by a set of weighted particles. The model takes into account only some parameters like battery model, electric powertrain, and vehicle dynamics. The development was validated through simulation including different driving situations. The proposed approach has as an objective to make contribution for generating reliable information regarding the RDR that could improve the quality of EV's driving assistance systems [8, 19]. A lot of scientist and engineers give more and more attention to state of function (SoF) estimation technology of power lithium ion batteries. In [20], a fuzzy c-mean clustering algorithm was developed in order to estimate the SoF of the EV's power lithium-ion battery. The fuzzy estimation is improved by the fuzzy c-mean algorithm taking into account at least three parameters as input (Battery state of charge, battery state of health, and charge-discharge rate). The authors conclude that experimental results demonstrate the advantage of the estimation model presented with the average error of estimation which approximate $8.69 \%$. In [21], a torque management strategy for pure electric vehicle based on fuzzy control is presented. The approach is based on an electric vehicle model-based AVL cruise. The authors has validated the approache and simulation results shows that fuzzy control torque management strategy improves the car's energy consumption.

We can note that one of the major fears of drivers is to break down due to the lack of energy before 
reaching their destination. However, as explained in the previous paragraph, many factors can influence the energy consumption of an electric vehicle. Existing models are impersonal. In this work, we are interested in energy optimization for electric vehicles using machine learning. The aim is to give priority to personal data in order to check whether the electric vehicle has enough energy to reach its destination before proposing the shortest route in terms of energy consumption.

\section{THE PROPOSED METHOD}

Existing models were based on both shared real-time data like traffic and mathematical model for energy consumption in order to calculate the optimal path. However, we know in the reality one of the big challenges is to estimate the energy consumption. It is very important to take into account the battery state-ofhealth and the particularities of each electric vehicle model. Furthermore, we know some journeys are regular, in particular home-to-work journeys.

In our approach, we will propose to consider historical data in order to predict the energy consumption of the journey. For that, we propose to use a machine learning and especially $k$-NN algorithm [22] which is a lazy learning. As input, we use the data composed of:

- IDDriver: It is a random number used in order to identify the driver. In order to respect the general data protection regulation (GDPR), this number is not associate with the real identity of the driver.

- Departure place: Corresponds to the current position of the vehicle.

- Destination place: It is the destination of the journey.

- Date: It is the date of the journey. We know the date can influence directly the traffic. In fact, the day before the holidays, the traffic is generally crowd.

- Time: Corresponds to the time of the journey. Like for the date, traffic can change considerably depending on the time. The return of the weekends, i.e., on Sundays at the end of the day, the traffic is heavier, especially on the highways.

- VehicleKind: It is the kind of the vehicle. The possible values are 0:car, 1:motorcycle, 2:truck, 3:bus, and 4:others. This parameter is very important because the consumption energy is totally different between car, motorcycle or truck.

- Traffic: This parameter is returned by web service RESTful.

- Weather: This parameter is returned by web service RESTful.

- Energy: This parameter represents the real consumption energy returned by the vehicle at the end of the journey.

When the driver want the optimize the consumption energy of his trip, he need to define his destination in mobile application. The latter will estimate the energy consumption by applying our proposed algorithm. The aim is, in first step, predict the energy consumption of the journey, and, in the second step, propose the optimal path in terms of energy consumption by using $A^{*}$ algorithm.

\subsection{Mathematical notation}

We represent the map by a directed graph $G(V, E)$ where $E$ represent the edges, i.e., the routes in the map and $\mathrm{V}$ represent the vertices i.e. the intersection between edges. We assume that for each edge an energy consumption $E_{c}: E \rightarrow \mathbf{R}$ is given. That represent the weight of each edge.

$A\left(x_{A}, y_{A}\right)$ is a vertex in $V$. The coordinates of $\mathrm{A}$ in the map are $\left(x_{A}, y_{A}\right) . A\left(x_{A}, y_{A}\right) \rightarrow B\left(x_{B}, y_{B}\right)$ represents one journey from point $\mathrm{A}$ with coordinate $A\left(x_{A}, y_{A}\right)$ to point $\mathrm{B}$ with coordinate $B\left(x_{B}, y_{B}\right)$. $N(A(x, y))$ represents the set of the neighbours of the point A. $N(A(x, y))=\left\{A_{i}\left(x_{i}, y_{i}\right) \in V \mid(x-50 m \leq\right.$ $\left.x_{i} \leq x+50 m\right)$ and $\left.\left(y-50 m \leq y_{i} \leq y+50 m\right)\right\} N_{\text {driver }}(A, B)$ represents the set of paths from $N(A(x, y))$ to $N(B(x, y))$ taken by the driver. $N_{\text {driver }}(A, B)=\left\{\forall u, v \in V, \exists\right.$ path from $u$ to $v$ where $u \in N_{\text {driver }}(A)$ and $\left.v \in N_{\text {driver }}(B)\right\}$. The predict of energy consumption of the journey noted $\hat{E}_{c}(A, B)$

\subsection{Proposed algorithm of estimation energy consumption}

The proposed algorithm illustrated in Figure 1 is based on $k$-NN algorithm. The aim is to estimate the consumption energy by calculating the average of the values of k nearest neighbours. If we do not have enough data, we use our mathematical model proposed in [6, 7]. 


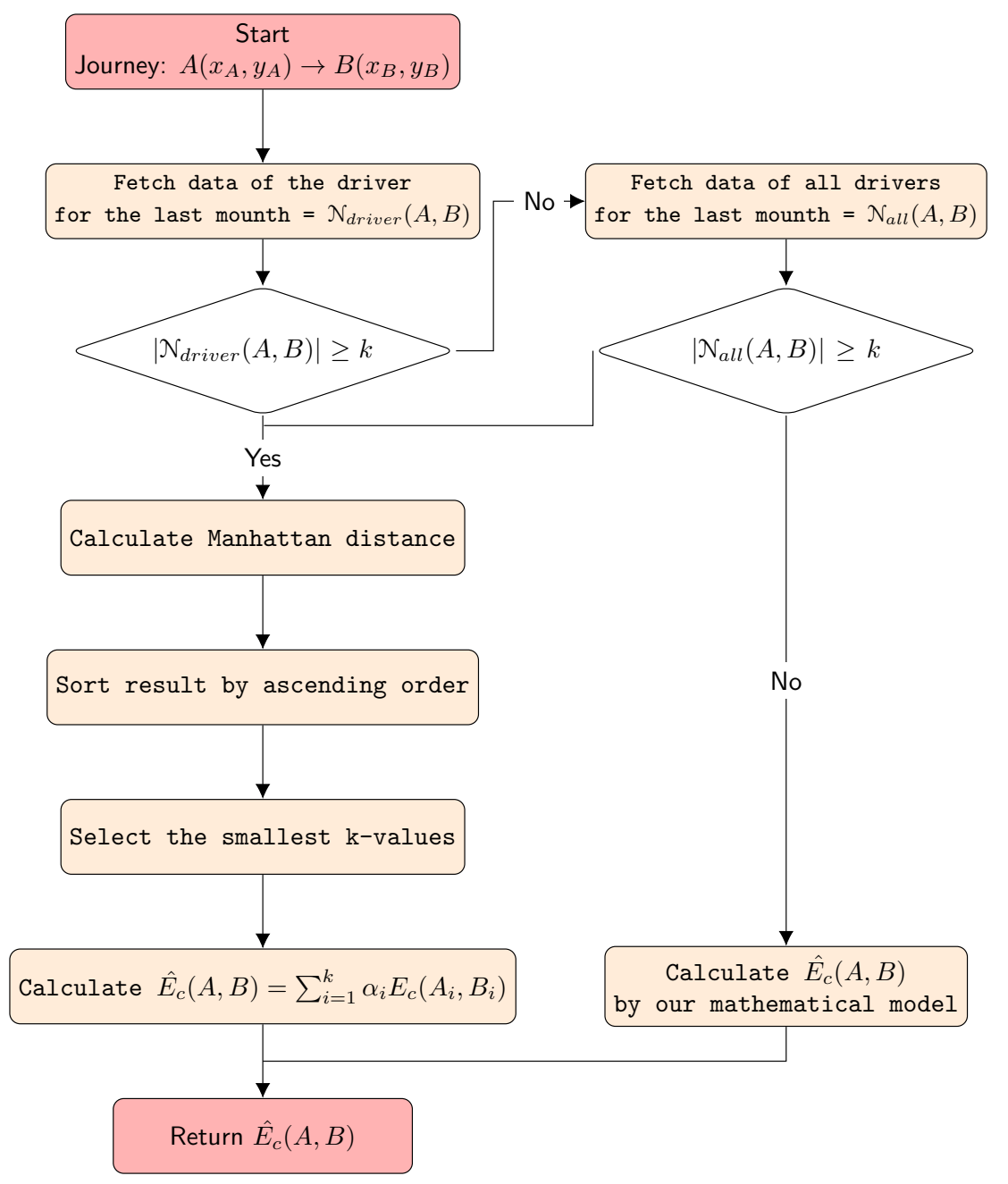

Figure 1. Algorithm proposed for the estimation of the energy consumption of the journey

The different steps are explained as:

- Let $\mathrm{A}$ the departure point with coordinates $\left(x_{A}, y_{A}\right)$ and $\mathrm{B}$ the destination point with coordinates $\left(x_{B}, y_{B}\right)$.

- Let $N(A(x, y))$ and $N(B(x, y)))$ the closest point from $A$ and $B$, respectively, and known in training observations

- We target all neighbor points from point $\mathrm{A}$ in the zone which is delimited by one square see Figure 2. The coordinates of upper left corner are $\left(x_{A}-50 m, y_{A}-50 m\right)$ and the coordinates of the down right corner are $\left(x_{A}+50 m, x_{A}+50 m\right)$. We do the same job for the point B.

(a) Fetch the data of the current driver by its ID for the last month

(b) If the number of fetched data is less than $\mathrm{k}$, fetch the data of all drivers

(c) Calculate the distance between the $\mathrm{B}$ destination point and fetched points in the target zone by using the Manhattan distance: $D_{m}(x, y)=\sum_{i=1}^{k}\left|x_{i}-y_{i}\right|$.

(d) Sort the calculated distance by ascending order

(e) Select the smallest k-values ,i.e., k-points

- If the number of fetched data is less than $\mathrm{k}$, return the estimated value calculated by our mathematical model presented in $[6,7]$, else return the predicted energy consumption $\hat{E}_{c}$ of the journey which is equal to: 


$$
\hat{E}_{c}(A, B)=\sum_{i=1}^{k} \alpha_{i} E_{c}\left(A_{i}, B_{i}\right)
$$

where, $E_{c}\left(A_{i}, B_{i}\right)$ is the function which fetch the energy consumption of $i^{t h}$-journey from point $A_{i}$ to point $B_{i}$. This value is fetched from training observations, and $k$ is the number of selected neighbor points. $\alpha_{i}$ represents the weight of the $i^{t h}$-journey where $\sum_{i=1}^{k} \alpha_{i}=1 \mathrm{We}$ assign the greater weight for the closer neighbors of the journey A-B than the neighbors which are further away.

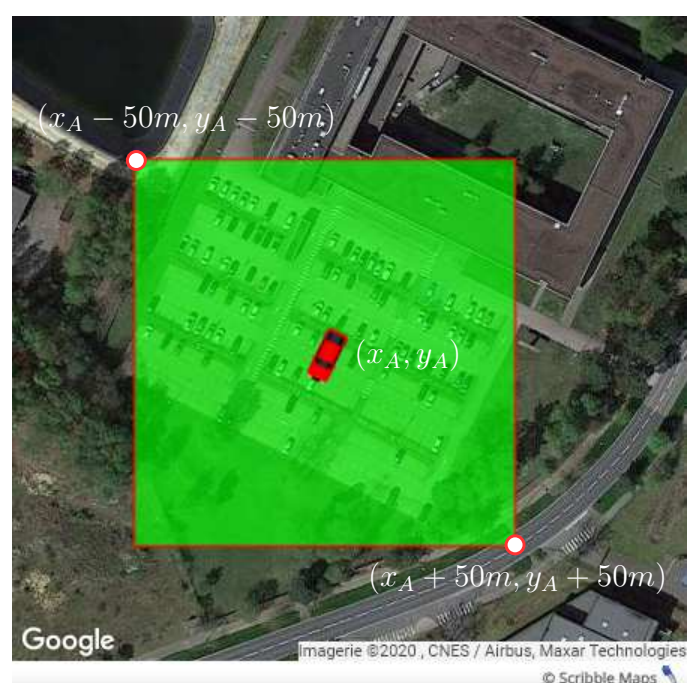

Figure 2. Neighbours vehicles perimeter

\subsection{Proposed algorithm of shortest path in terms of consumption energy}

In order to propose the optimal path on terms of energy consumption, we were inspired by the $\mathrm{A}^{*}$ algorithm. The used cost function in (2) is composed of two terms. The first term $t(n)$ which represents the real cost value to reach node $n$. The second term $h(n)$ represents the estimation of energy consumption calculated by our proposed algorithm.

$$
f(n)=t(n)+h(n)
$$

Our shortest path algorithm (SPA) will also maintain two lists, same with $A^{*}$ algorithm, openlist and closelist. Nodes in openlist are nodes which are going to be visited and nodes in closelist are nodes which have been visited. The whole process is detailed as:

- Add the departure point into the openlist;

- Repeat the following process:

(a) Traverse the openlist to find the node $u$ with the smallest estimated value

(b) Delete $u$ from openlist and add to the closelist

(c) Traverse the connected nodes $v$. If $v$ is in the closelist, ignore it. If $v$ is not in the openlist, add it to the openlist (add a pointer to the node from). If $v$ is in openlist, check whether the estimation value is smaller than the previous one. If so, update the value.

- If node $u$ is the destination node, break the loop. If openlist is empty, break the loop. In this case, it means that the destination node is unreachable from the departure node.

We are about to use the heuristic estimation function of the energy consumption model in which the $h(n)$ value is the energy consumption between the departure node and the destination node and the value of 
$t(n)$ is the energy already consumed from the departure node to the current node. This is also a kind of the best-first algorithm which select always the greedy strategy in each step.

\section{RESULTS AND DISCUSSION}

In order to evaluate our proposed model, we use the simulator simulation of urban mobility (SUMO) [23]. We choose to simulate the traffic around our laboratory. We can see in the Figure 3 the map used for our simulation. In the Figure 4, we illustrate the coordinates of departure and destination points. The default model of electric vehicle energy consumption [24], provided by SUMO, is used. The weather is not considered in our simulation because this parameter is not implemented in SUMO.

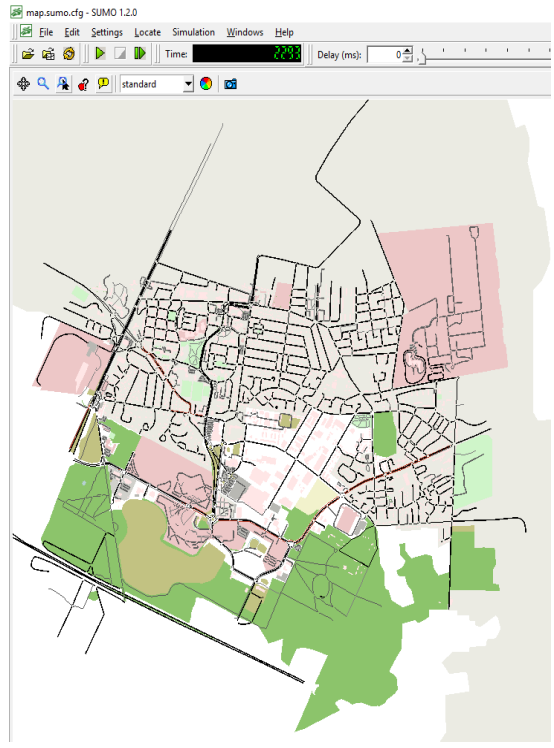

Figure 3. Map used in our simulation

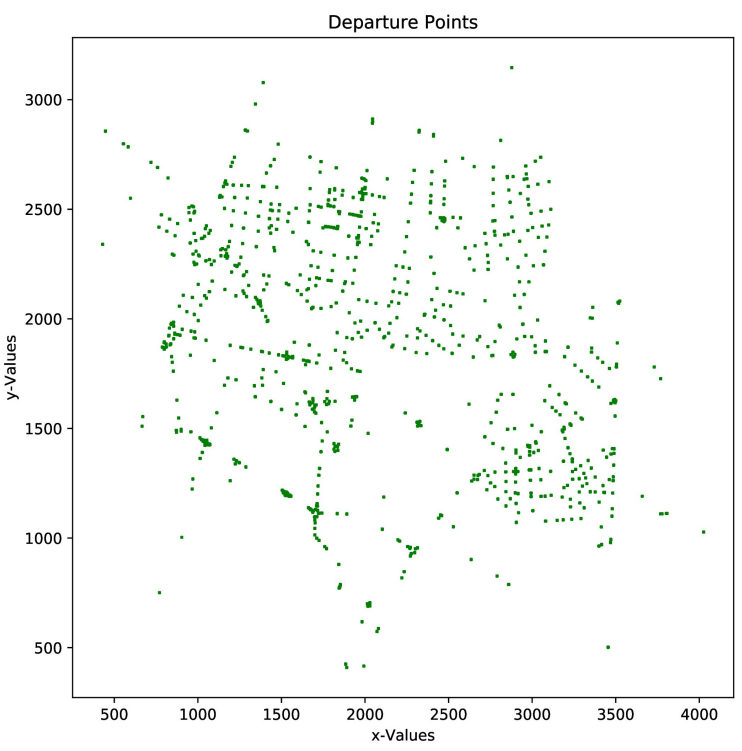

Figure 4. Departure and arrived points in map

In this simulation, the departure and the destination point are taken totally randomly. The used training data set are composed of 12000 journeys which represent $70 \%$ of data. In each entry of the training data set, we have several parameters, including vehicle id, coordinate of the departure and destination point, departure time, traffic situation indicator, vehicle type, total trip distance, energy consumption and duration of the trip. We call the process from the vehicle start at the departure point until the vehicle stops at the destination as a trip.

The first step is to generate the training data. First of all, in the part of simulating the traffic flow, we use the RandomTrips script provided by SUMO, and the generated vehicle behavior conforms to the Poisson distribution. We estimate that as a possible traffic flow situation. Then we add EV in the traffic. The departure and destination positions are randomly distributed. The vehicle mass, the maximum battery capacity, the maximum power, the front surface area, the air drag coefficient, the radial drag coefficient, the rolling resistance coefficient, recuperation and drive efficiency and other parameters remains the same. A given time slot, a certain number of EV are added to the traffic. Here, we assume that the number of newly added EVs is relatively small compared to the original traffic flow in the map.

During the simulation of EV driving, we need to record the three parameters of the travel distance, duration of the trip and vehicle's energy consumption until the vehicle reaches the destination point. At the same time, we also have the other 6 parameters: vehicle id, coordinate of the departure and destination point, departure time, traffic situation indicator, vehicle type. We define the waiting time: if the speed of the EV in the current time step is less than $0.1 \mathrm{~m} / \mathrm{s}$, then the vehicle is considered as in the waiting state. The waiting time is calculated from the last time when the EV speed is greater than $0.1 \mathrm{~m} / \mathrm{s}$ until the next time when the vehicle's speed is greater than $0.1 \mathrm{~m} / \mathrm{s}$.

The second step is to use our proposed algorithm (based on $k-\mathrm{NN}$ ) of estimation consumption energy. 
We use the sklearn library [25] in order to implement our algorithm. The core process has been described before. We just need to provide the input parameters which are the coordinates of departure and destination point, departure time which is usually the current time, traffic situation indicator and the vehicle type which is mostly the electric car in our case. Then, we can obtain the estimated value of the energy consumption $\hat{E} c$. By changing the value of $K$, we can achieve different estimation results with different accuracy. In Figure 5, we present the explained variance of the test data by picking different $k$-value from 1 to 10 to find that the best choice of the $k$-value is 5 which is equal to 0.964529 . The closer the value is to 1 , the more accurate the model is. Also, we can see in Figure 6 the root mean square error in function of $k$-values.

We can see the distribution of test data in Figure 7. Each point in the curve represents one journey. The $x$-value represent the real energy consumption (obtained by simulation) and the $y$-value represents the estimated energy consumption obtained by our proposed algorithm.

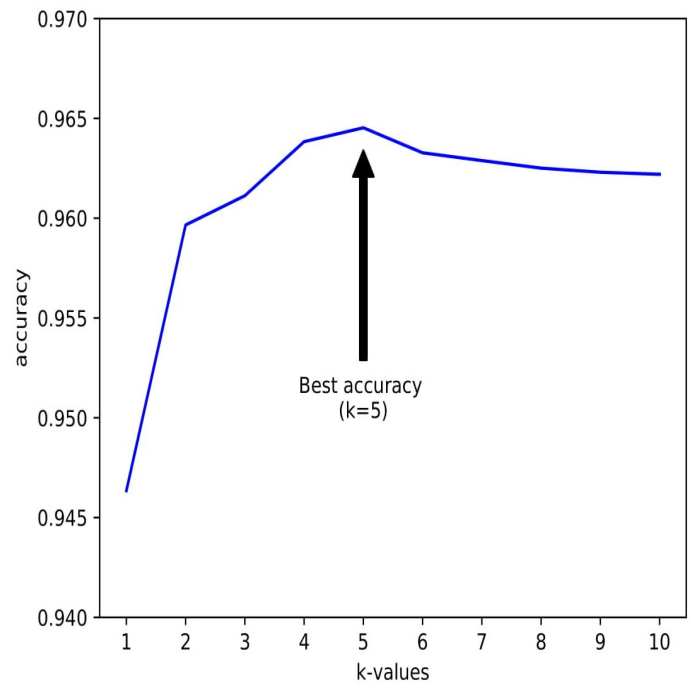

Figure 5. Variance regression score in function of $k$-values

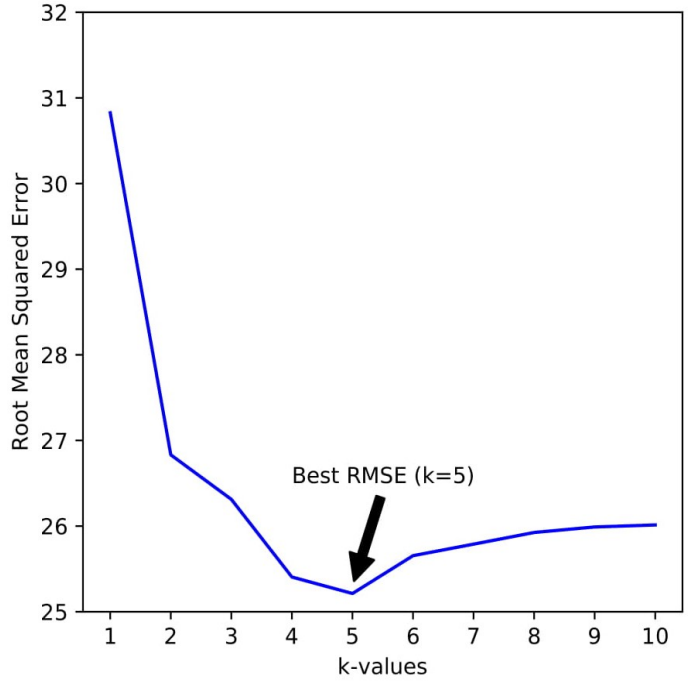

Figure 6. Root mean square error in function of $k$-values

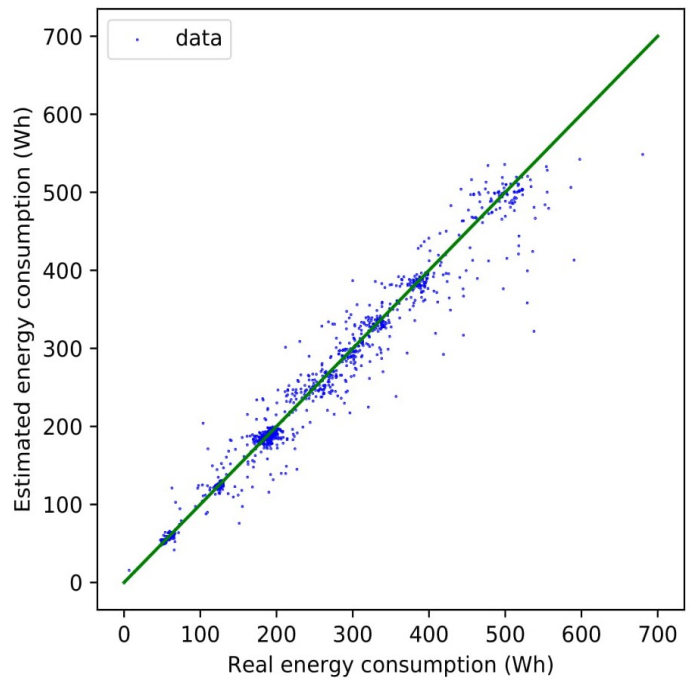

Figure 7. Distribution of energy consumption estimated for $k=5$ 
The third step is to apply the proposed algorithm of estimation energy consumption (with $k$-value determined by the previous step) to the algorithm of shortest path. We just need to modify the heuristic function part. Usually, the Euclidean distance between the departure point and the destination point for the heuristic estimation function is used. In order to take into account the energy consumption model, we replace the heuristic estimation function with calculating the energy consumption between the departure point and the destination point. It should be noted that the value of $t(n)$ in the cost function will also changed to the energy already consumed.

To find the best value of $k$ in the proposed algorithm of energy consumption estimation, we have randomly selected the departure and destination points as the test data for simulation. At the same time, we use our proposed algorithm to estimate the test data in order to calculate the accuracy of the estimation results. By changing the value of $K$, we can achieve different estimation results with different accuracy. In Figure 5, we present the result of the test data by picking different $k$ value from 1 to 10 to find that the best choice of the $k$-value is 5 .

\section{CONCLUSION}

In the literature, many studies and approaches have been presented to estimate and optimise the EV energy-consumption. Some of them consider only a single factor that influence EV energy consumption, while on the other hand they consider all factors which could be significant in the EV energy consumption. Offering the most accurate estimation of energy consumption is a major point if we want to convince more consumers to switch to EV. Several factors impact this consumption and are difficult to model. In this paper, we have provided a solution based on lazy learning approach in order to obtain the best estimation of energy consumption and to propose the optimal path to reach a destination. The obtained results achieved using simulation tools are very promising. As a future direction, firstly, we plan to carry out full-scale tests in order to verify the relevance of the obtained results. Secondly, we should improve the quality of the model developed by investigating artificial intelligence-based solutions and particularly deep learning. To do so, we have designed a deep learning vision model under hybrid datasets (publicly available datasets combined with our own datasets acquired in real traffic conditions) for smart and secure mobility. We will integrate and adapt them to build and train a model for energy consumption in order to have a full platform in which both security and energy-consumption optimisation will be considered as a two main factors in the future EV. Therefore, within the same deep learning architecture, we propose to incorporate the relationship between all parameters related to environment, driver behaviour, and vehicle model.

\section{REFERENCES}

[1] K. Hammoudi, et al., "Towards a model of car parking assistance system using camera networks: Slot analysis and communication management," Proc. IEEE 20th Int. Conf. High Performance Computing and Communications; IEEE 16th Int. Conf. Smart City; IEEE 4th Int. Conf. Data Science and Systems (HPCC/SmartCity/DSS), 2018, pp. 1248-1255.

[2] M. Randriamasy, et al., "Reliable Vehicle Location in Electronic Toll Collection Service with Cooperative Intelligent Transportation Systems," 28th Annual IEEE International Symposium on Personal, Indoor and Mobile Radio Communications (IEEE PIMRC 2017), 2019, pp. 1-7.

[3] M. Randriamasy, A. Cabani, H. Chafouk, G. Fremont, "Geolocation Process to Perform the Electronic Toll Collection Using the ITS-G5 Technology," IEEE Transactions on Vehicular Technology, vol. 68, no. 9, pp. 8570-8582, 2019.

[4] M. Randriamasy, A. Cabani, H. Chafouk, G. Fremont, "Formally validated of novel tolling service with the its-g5," IEEE Access, vol. 7, pp. 41133-41144, 2019.

[5] ETSI TR, "Intelligent Transport Systems (ITS) - PreStandardization Study on payment applications in Cooperative ITS using V2I communication,” ETSI TR 103579 v1.1.1 2020-09, 2020.

[6] A. Cabani, R. Khemmar, J.-Y. Ertaud, J. Mouzna, "Intelligent navigation system-based optimization of the energy consumption," 2015 IEEE Intelligent Vehicles Symposium (IV), pp. 785-789, 2015.

[7] A. Cabani, R. Khemmar, J.-Y. Ertaud, R. Rossi, X. Savatier, "ADAS multi-sensor fusion system-based security and energy optimisation for an electric vehicle," International Journal of Vehicle Autonomous Systems, Inderscience Publishers, vol. 14, no. 4, pp. 345-366, 2019. 
[8] B. O. N. Varga, A. Sagoian, F. Mariașiu, "Prediction of electric vehicle range: A comprehensive review of current issues and challenges," Energies, vol. 12, no 5, pp. 1-10, 2019.

[9] S.M. Rezvanizaniani, Z. Liu, Y. Chen, Y., J. Lee, "Review and recent advances in battery health monitoring and prognostics technologies for electric vehicle (EV) safety and mobility," Journal of Power Sources, vol. 256, pp. 110-124, 2014.

[10] K. Sentoff, L. Aultman-Hall, B. A. Holmén, "Implications of driving style and road grade for accurate vehicle activity data and emissions estimates," Transportation Research Part D: Transport and Environment, vol. 35, pp. 175-188, 2015.

[11] X. Wu, X. He, G. Yu, A. Harmandayan and Y. Wang, "Energy-Optimal Speed Control for Electric Vehicles on Signalized Arterials," in IEEE Transactions on Intelligent Transportation Systems, vol. 16, no. 5, pp. 2786-2796, Oct. 2015.

[12] T. Yuksel, J.J. Michalek, "Effects of regional temperature on electric vehicle efficiency, range, and emissions in the United States," Environmental Science and Technology, vol. 49, pp. 3974-3980, 2015.

[13] J. Wang, I. Besselink, H. Nijmeijer, "Battery electric vehicle energy consumption prediction for a trip based on route information," Proceedings of the Institution of Mechanical Engineers, Part D: Journal of Automobile Engineering, vol. 232, no. 11, 2018, pp. 1528-1542.

[14] T. Bär, D. Nienhüser, R. Kohlhaas and J. M. Zöllner, "Probabilistic driving style determination by means of a situation based analysis of the vehicle data," 2011 14th International IEEE Conference on Intelligent Transportation Systems (ITSC), Washington, DC, 2011, pp. 1698-1703.

[15] B. D. Ziebart, A. Maas, J. A. Bagnell, AnA.ind K. Dey, "Maximum entropy inverse reinforcement learning," in Proceedings of the 23rd national conference on Artificial intelligence-Volume 3 (AAAI'08), AAAI Press, 2008, pp. 1433-1438, 2008.

[16] D. Delling, A. V. Goldberg, T. Pajor, R. F. Werneck, "Customizable route planning in road networks," Transportation Science, vol. 51, no. 2, pp. 566-591, 2015.

[17] M. Baum, J. Dibbelt, T. Pajor, D. Wagner, "Energy-optimal routes for electric vehicles," in Proceedings of the 21st ACM SIGSPATIAL International Conference on Advances in Geographic Information Systems, Orlando, FL, USA, 5-8 November 2013, 2013, pp. 54-63.

[18] OpenStreetMap Contributors. Planet Dump. 2017. [online]. Available : https://www.openstreetmap.org.

[19] J. A. Oliva, C. Weihrauch and T. Bertram, "Model-based remaining driving range prediction in electric vehicles by using particle filtering and Markov chains," 2013 World Electric Vehicle Symposium and Exhibition (EVS27), Barcelona, pp. 1-10, 2013.

[20] D. Wang, F. Yang, L. Gan, Y. Li, "Fuzzy Prediction of Power Lithium Ion Battery State of Function Based on the Fuzzy c-Means Clustering Algorithm," World Electric Vehicle Journal, vol. 10, no. 1, pp. 1-13, 2019.

[21] S. Jikang, C. Lingshan, "Torque Management Strategy of Pure Electric Vehicle Based On Fuzzy Control," International Journal of Research in Engineering and Science (IJRES), vol 6, no. 4, pp. 1-9, 2018.

[22] N.S. Altman, "An Introduction to Kernel and Nearest-Neighbor Nonparametric Regression," The American Statistician, vol. 46, no. 3, pp. 175-185, 1992.

[23] Daniel Krajzewicz, Jakob Erdmann, Michael Behrisch, and Laura Bieker, "Recent Development and Applications of SUMO - Simulation of Urban MObility," International Journal On Advances in Systems and Measurements, vol. 5, no. 3\&4, pp. 128-138, Dec 2012.

[24] T. Kurczveil, P.A. López, E. Schnieder, "Implementation of an Energy Model and a Charging Infrastructure in SUMO," In Behrisch, M., Krajzewicz, D., Weber, M. (eds.) Simulation of Urban Mobility, Lecture Notes in Computer Science, vol. 8594, pp. 33-43, 2014.

[25] F. Pedregosa, et al., "Scikit-learn: Machine Learning in Python," Journal of Machine Learning Research, vol. 12, pp. 2825-2830, 2011. 